COMMUNICATIONS IN

ANALYSIS AND GEOMETRY

Volume 12, Number 3, 487-510, 2004

\title{
Existence and Compactness of Minimizers of the Yamabe Problem on Manifolds with Boundary
}

\author{
Henrique Araúso
}

\begin{abstract}
Dedicated to José Escobar (in memoriam).
We show existence of minimizers of the Yamabe functional on a compact Riemannian manifold with boundary $(M, g)$, of dimension $n \geq 3$, restricted to the set of all metrics conformal to $g$ and satisfying $a V+b A=1$, where $V$ and $A$ are the volume of $M$ and area of $\partial M$, respectively, when $a$ and $b$ are positive real numbers and when the infimum of the functional on that set is stricly less than the corresponding quantity on the standard Euclidean half-sphere. This shows that for such manifolds we can deform $g$ conformally to obtain a metric with constant scalar curvature $R$ and constant mean curvature $h$ on the boundary which are related by $b R=2 n h a$. These results are already known when $(M, g)$ is locally conformally flat or when $n \geq 5$ and $\partial M$ is not umbilic. They extend for arbitrary positive $a$ and $b$ results known for the case when $a=1, b=0$, the case when $a=0, b=1$, and the case when $b$ is small. We also show a compactness result for the set of all minimizers when the metric is allowed to vary on a small neighborhood of a given base metric satisfying the above condition.
\end{abstract}

\section{Introduction.}

The Yamabe problem for manifolds without boundary asked whether a smooth compact closed Riemannian manifold $M$ of dimension $n \geq 3$, with Riemannian metric $g$, admitted a Riemannian metric $\tilde{g}$ conformal to $g$ having constant scalar curvature. The Yamabe problem was solved in the affirmative after contributions by Yamabe $([\mathrm{Y}])$, Trudinger $([\mathrm{T}])$, Aubin $([\mathrm{Au}])$ and Schoen ([S]). A survey on the Yamabe problem for manifolds without boundary can be found in [LP]. In 1992, Escobar ([E1]) showed that almost any compact Riemannian manifold with boundary $M$ of dimension $n \geq 3$, with Riemannian metric $g$, admits a smooth Riemannian metric $\tilde{g}$ conformal to $g$

\footnotetext{
${ }^{1} 2000$ Mathematics Subject Classification. Primary: 58C21, 53J32.
} 
having constant scalar curvature and minimal boundary. Thus, he provided an affirmative answer to an extension of the Yamabe problem to manifolds with boundary in the cases considered. The metric $\tilde{g}=u^{\frac{4}{n-2}} g$ has constant scalar curvature and minimal boundary if and only if $u$ is a smooth positive solution to

$$
\left\{\begin{array}{lr}
\Delta u-\frac{n-2}{4(n-1)} R u+\frac{2 n}{n-2} \lambda u^{\frac{n+2}{n-2}}=0 & \text { on } M, \\
\frac{\partial u}{\partial \eta}+\frac{n-2}{2} h u=0 & \text { on } \partial M,
\end{array}\right.
$$

where $\Delta$ is the Laplace operator induced by the metric $g, \eta$ is the unit outward normal on $\partial M$ with respect to $g, R$ is the scalar curvature of $g$, $h$ is the mean curvature of $g$ on $\partial M$ and $\lambda$ is a Lagrange multiplier. The above equation turns out to be the Euler-Lagrange Equation associated with nonnegative critical points of the functional

$$
E(u)=\int_{M}|\nabla u|^{2} d \omega+\frac{n-2}{4(n-1)} \int_{M} R u^{2} d \omega+\frac{n-2}{2} \int_{\partial M} h u^{2} d \sigma,
$$

when restricted to the constraint set $B=\left\{u \in H^{1}(M) \mid \int_{M} u^{\frac{2 n}{n-2}} d \omega=1\right\}$, where $H^{1}(M)$ is the Sobolev space of square integrable functions with square integrable weak first derivatives, and $d \omega$ is the volume element induced by $g$. The resolution of the Yamabe problem is then achieved by showing existence of a smooth positive critical point of the functional $E$ restricted to the above constraint set. This is typically done in two steps. First the exponent $\frac{n+2}{n-2}$ in Equation 1 is lowered by finding minimizers of the functional $E$ on the constraint sets $B_{\alpha}=\left\{u \in H^{1}(M) \mid \int_{M} u^{\alpha} d \omega=1\right\}, \alpha<\frac{2 n}{n-2}$. Existence of such minimizers is a consequence of the compactness of the Sobolev Embedding $H^{1}(M) \hookrightarrow L^{\alpha}(M)$, for $\alpha$ strictly less than $\frac{2 n}{n-2}$. A minimizer $u_{\alpha}$ of the functional $E$ on the set $B_{\alpha}$ is a (weak) solution to

$$
\left\{\begin{array}{lr}
\Delta u_{\alpha}-\frac{n-2}{4(n-1)} R u_{\alpha}+\frac{2 n}{n-2} \lambda u_{\alpha}^{\alpha-1}=0 & \text { on } M \\
\frac{\partial u_{\alpha}}{\partial \eta}+\frac{n-2}{2} h u_{\alpha}=0 & \text { on } \partial M .
\end{array}\right.
$$

A regularity result by Cherrier $([\mathrm{C}])$ implies that any weak solution of Equation 2 is smooth. The second step consists in using elliptic estimates to show that the set of all minimizers $u_{\alpha}, \alpha<\frac{2 n}{n-2}$ and sufficiently close to $\frac{2 n}{n-2}$, is uniformly bounded in the $C^{2, \gamma}$ norm, for some $0<\gamma<1$. A sequence of $u_{\alpha}$ 's will then converge strongly in the $C^{2}$ norm to a minimizer of the functional $E$ on the set $B$. A regularity result by Cherrier again 
shows that such a minimizer is smooth, and an application of the Maximum Principle for Elliptic Operators to Equation 1 shows that it is positive. Let $Q(M, g)=\inf \{E(u) \mid u \in B\}$, and let $Q\left(S_{+}^{n}\right)$ be the corresponding quantity when $M$ is the $n$-dimensional upper half sphere in Euclidean space with the standard metric. We always have that $Q(M, g) \leq Q\left(S_{+}^{n}\right)$. In order to kick start the bootstrapping argument used in obtaining the desired $C^{2, \gamma}$ norm bounds, we must impose the condition that $Q(M, g)<Q\left(S_{+}^{n}\right)$. Indeed, such uniform bounds cannot be found on $S_{+}^{n}$ due to the noncompactness of its group of conformal diffeomorphisms. The bulk of [E1] is devoted to showing that $Q(M, g)<Q\left(S_{+}^{n}\right)$ is satisfied for a large class of manifolds with boundary.

When $u$ is smooth and positive and $\tilde{g}=u^{\frac{4}{n-2}} g$, then the condition $\int_{M} u^{\frac{2 n}{n-2}} d \omega=1$ is equivalent to requiring that the volume of $(M, \tilde{g})$ is unitary. We may also choose as our constraint set the set of metrics conformal to a base metric $g$ satisfying $a V+b A=1$, where $V$ is the volume of $M, A$ is the area of $\partial M$ and $a, b$ are real parameters with $a \geq 0$ (when $a=0$ we choose $b=1$ ). This will correspond to the set $B^{a, b}=\left\{u \in H^{1}(M) \mid a \int_{M} u^{\frac{2 n}{n-2}} d \omega+\int_{\partial M} u^{\frac{2(n-1)}{n-2}} d \sigma=1\right\}$, where $d \sigma$ is the area element on $\partial M$ induced by $g$. If we let $Q^{a, b}(M, g)=\inf \left\{E(u) \mid u \in B^{a, b}\right\}$, then $Q^{a, b}(M, g) \leq Q^{a, b}\left(S_{+}^{n}\right)$, where $Q^{a, b}\left(S_{+}^{n}\right)$ is the corresponding quantity when $M$ is the $n$-dimensional upper half sphere in Euclidean space with the standard metric ([E4]). A positive critical point $u$ of the functional $E$ restricted to the set $B^{a, b}$ is a (necessarily smooth) solution of

$$
\left\{\begin{array}{lr}
\Delta u-\frac{n-2}{4(n-1)} R u+\frac{2 n}{n-2} \lambda a u^{\frac{n+2}{n-2}}=0 & \text { on } M, \\
\frac{\partial u}{\partial \eta}+\frac{n-2}{2} h u=\frac{2(n-1)}{n-2} \lambda b u^{\frac{n}{n-2}} & \text { on } \partial M,
\end{array}\right.
$$

where $\lambda$ is a Lagrange multiplier. Equation 3 says that the metric $\tilde{g}=u^{\frac{4}{n-2}}$ has constant scalar curvature $\tilde{R}=\frac{8 n(n-1) \lambda a}{(n-2)^{2}}$ and constant mean curvature $\tilde{h}=\frac{4(n-1) \lambda b}{(n-2)^{2}}$. When $a>0$, existence of such critical points is proved in [E4] for manifolds of nonpositive type $\left(Q^{a, b}(M, g) \leq 0\right)$ and for almost any manifold of positive type if $b$ is sufficiently small. The case $a=0, b=1$ is treated in [E2]. In [HL1] and [HL2], Han and Li showed existence of solutions to Equation 3 when $a>0,(M, g)$ is of positive type and either $(M, g)$ is locally conformally flat with umbilic boundary or $n \geq 5$ and $\partial M$ has a nonumbilic point. In the same cases and when $(M, g)$ is not conformally equivalent to $S_{+}^{n}$, they also show compactness results (uniform bounds on $C^{2}$ norms) for the set of all solutions (after normalization). 
In Section 2 of this paper we show existence of minimizers of the functional $E$ on $B^{a, b}$ when $a>0, b>0$ and $Q^{a, b}(M, g)<Q^{a, b}\left(S_{+}^{n}\right)$, following the two step program indicated above. In particular this shows existence of solutions to Equation 3 in that case. It seems safe to conjecture that the strict inequality $Q^{a, b}(M, g)<Q^{a, b}\left(S_{+}^{n}\right)$ will hold under conditions similar to those found in [E2] or [HL1] and [HL2]. This will be addressed in a forthcoming paper. It is not known whether equality holds only if the manifold is globally conformal to the standerd sphere. In Section 3 we establish compactness results for the set of all minimizers when the base metric is allowed to vary on a small $C^{3}$ neighborhood in the space of Riemannian metrics. In this Section we also consider the case $a=0, b=1$. We assume finiteness of the first eigenvalue of the problem

$$
\begin{cases}\Delta u-\frac{n-2}{4(n-1)} R u=0 & \text { on } M, \\ \frac{\partial u}{\partial \eta}+\frac{n-2}{2} h u=\lambda u & \text { on } \partial M\end{cases}
$$

(see [E3]) and we show that the critical points found in [E2] are indeed minimizers, and compactness results are established.

\section{Existence of minimizers.}

Let $M$ be a compact Riemannian manifold with boundary, of dimension $n \geq 3$. Let $g$ be a fixed smooth Riemannian metric on $M$. Let $[g]=$ $\left\{u^{\frac{4}{n-2}} g \mid u>0, u \in C^{\infty}\right\}$ be the conformal class of $g$. Let $R$ denote the scalar curvature of $g$ and let $h$ denote its mean curvature on $\partial M$. Let $\tilde{R}$, $\tilde{h}$ respectively denote the scalar and mean curvatures of the Riemannian metric $\tilde{g}=u^{\frac{4}{n-2}} g \in[g]$. $\tilde{R}$ and $\tilde{h}$ are related to $R$ and $h$ by

$$
\left\{\begin{array}{lr}
\Delta u-\frac{n-2}{4(n-1)} R u+\frac{n-2}{4(n-1)} \tilde{R} u^{\frac{n+2}{n-2}}=0 & \text { on } M, \\
\frac{\partial u}{\partial \eta}+\frac{n-2}{2} h u=\frac{n-2}{2} \tilde{h} u^{\frac{n}{n-2}} & \text { on } \partial M,
\end{array}\right.
$$

where $\Delta$ is the Laplacian induced by $g$ and $\eta$ is the unit outward normal on $\partial M$ determined by $g$. Let $p=\frac{2 n}{n-2}$ and $q=\frac{2(n-1)}{n-2}$. The volume of $(M, \tilde{g})$ is given by $\int_{M} u^{p} d \omega$, where $d \omega$ is the volume element induced by $g$, and the area of $(\partial M, \tilde{g})$ is given by $\int_{\partial M} u^{q} d \sigma$, where $d \sigma$ is the area element induced by $g$. For $a, b>0$ let us define

$$
B_{p, q, g}^{a, b}=\left\{u \in H^{1}(M) \mid a \int_{M} u^{p} d \omega+b \int_{\partial M} u^{q} d \sigma=1\right\},
$$


where $H^{1}(M)$ is the space of weakly differentiable $L^{2}$ functions with weak derivatives in $L^{2}$. For $u \in H^{1}(M)$, we define

$$
E(u, g)=\int_{M}|\nabla u|^{2} d \omega+\frac{n-2}{4(n-1)} \int_{M} R u^{2} d \omega+\frac{n-2}{2} \int_{\partial M} h u^{2} d \sigma,
$$

where $\nabla u$ is the gradient of $u$ with respect to $g$. Nonnegative critical points of the functional $E$ restricted to $B_{p, q, g}^{a, b}$ correspond to weak solutions of

$$
\left\{\begin{array}{lr}
\Delta u-\frac{n-2}{4(n-1)} R u+\lambda a p u^{p-1}=0 & \text { on } M, \\
\frac{\partial u}{\partial \eta}+\frac{n-2}{2} h u=\lambda b q u^{q-1} & \text { on } \partial M,
\end{array}\right.
$$

where

$$
\lambda=\frac{E(u)}{a p \int_{M} u^{p} d \omega+b q \int_{\partial M} u^{q} d \sigma} .
$$

A result by Cherrier $([\mathrm{C}])$ says that a weak solution of Equation 7 is smooth. The Maximum Principle for Elliptic Operators implies that a nonnegative smooth solution must either be positive or vanish identically. We define

$$
Q_{p, q}^{a, b}(g)=\inf \left\{E(u, g) \mid u \in H^{1}(M), u \in B_{p, q, g}^{a, b}\right\}
$$

We note that we may restrict ourselves to nonegative functions $u$ in the definition of $Q_{p, q}^{a, b}(g)$ without affecting the result. We always have that $-\infty<$ $Q_{p, q}^{a, b}(g) \leq Q_{p, q}^{a, b}\left(S_{+}^{n}\right)$, where $S_{+}^{n}$ is the upper half sphere in Euclidean space with the standard metric (see [E4]). In this section we will be mostly dealing with one fixed metric on $M$, and we will write $B_{p, q}^{a, b}, E(u)$ and $Q_{p, q}^{a, b}$ except when confusion may arise. To fix terminology, we will say that $u$ is a $Q_{p, q}^{a, b}$ minimizer if $u \in B_{p, q}^{a, b}$ and $E(u)=Q_{p, q}^{a, b}$. If $u$ is a $Q_{p, q}^{a, b}$ minimizer then it does not change sign and $|u|$ is a smooth positive solution of Equation 7, with $\lambda=\frac{Q_{p, q}^{a, b}}{a p \int_{M}|u|^{p} d \omega+b q \int_{\partial M}|u|^{q} d \sigma}$. In this section we prove the following theorem.

Theorem 2.1. Suppose that $Q_{p, q}^{a, b}<Q_{p, q}^{a, b}\left(S_{+}^{n}\right)$. Then there exists a $Q_{p, q}^{a, b}$ minimizer. Equation 7 has a smooth positive solution $u$ with $\lambda=$ $\frac{Q_{p, q}^{a, b}}{a p \int_{M} u^{p} d \omega+b q \int_{\partial M} u^{q} d \sigma}$, and $\tilde{g}=u^{\frac{4}{n-2}} g$ is a smooth Riemannian metric with constant scalar curvature $\tilde{R}=\frac{4(n-1)}{n-2} \lambda a p$ and constant mean curvature $\tilde{h}=\frac{2}{n-2} \lambda b q$. 
Clearly, only the statement about the existence of a $Q_{p, q}^{a, b}$ minimizer needs to be proved. Following a standard argument, we first lower the exponents in Equation 7 by way of the following definitions. For $2 \leq \alpha \leq p, 2 \leq \beta \leq q$, we let

$$
B_{\alpha, \beta}^{a, b}:=\left\{\left.u \in H^{1}(M)\left|a \int_{M}\right| u\right|^{\alpha} d \omega+b \int_{\partial M}|u|^{\beta} d \sigma=1\right\}
$$

and

$$
Q_{\alpha, \beta}^{a, b}:=\inf \left\{E(u) \mid u \in B_{\alpha, \beta}^{a, b}\right\} .
$$

It follows from Hölder's Inequality that $Q_{\alpha, \beta}^{a, b}$ is finite. When $2 \leq \alpha<p$, $2 \leq \beta<q$, it is standard to show, using the compactness of the embeddings $H^{1}(M) \hookrightarrow L^{\alpha}(M)$ and $H^{1}(M) \hookrightarrow L^{\beta}(\partial M)$, that there exists $u \in B_{\alpha, \beta}^{a, b}$ such that $E(u)=Q_{\alpha, \beta}^{a, b}$. We will say that such $u$ is a $Q_{\alpha, \beta}^{a, b}$ minimizer. As in the case when $\alpha=p, \beta=q$, a $Q_{\alpha, \beta}^{a, b}$ minimizer is smooth and does not change sign. We will denote by $u_{\alpha, \beta}$ any positive $Q_{\alpha, \beta}^{a, b}$ minimizer. A positive $Q_{\alpha, \beta}^{a, b}$ minimizer $u_{\alpha, \beta}$ satisfies the Euler-Lagrange equation

$$
\left\{\begin{array}{lr}
\Delta u_{\alpha, \beta}-\frac{n-2}{4(n-1)} R u_{\alpha, \beta}+a \alpha \lambda_{\alpha, \beta} u_{\alpha, \beta}^{\alpha-1}=0 & \text { on } M, \\
\frac{\partial u_{\alpha, \beta}}{\partial \eta}+\frac{n-2}{2} h u_{\alpha, \beta}=b \beta \lambda_{\alpha, \beta} u_{\alpha, \beta}^{\beta-1} & \text { on } \partial M,
\end{array}\right.
$$

where

$$
\lambda_{\alpha, \beta}=\frac{Q_{\alpha, \beta}^{a, b}}{a \alpha \int_{M} u_{\alpha, \beta}^{\alpha} d \omega+b \beta \int_{\partial M} u_{\alpha, \beta}^{\beta} d \sigma} .
$$

We will show that a sequence $u_{\alpha_{i}, \beta_{i}}$ converges in $C^{2}$ norm to a $Q_{p, q}^{a, b}$ minimizer $u$ as $\left(\alpha_{i}, \beta_{i}\right)$ tends to $(p, q)$. This will be done in two steps. First we show that suitable $L^{r}(M)$ and $L^{s}(\partial M)$ norms are uniformly bounded for the $u_{\alpha, \beta}$, for $(\alpha, \beta)$ sufficiently close to $(p, q)$. This is the content of Proposition 2.6. Next we use elliptic regularity to show that the $C^{2, \gamma}$ norms $(0<\gamma<1)$ of the $u_{\alpha, \beta}$ are also uniformly bounded (Proposition 2.10). To prove Proposition 2.6 we need a Sobolev type inequality which in a sense holds uniformly for all compact manifolds. This is Lemma 2.5. As the level set $B_{p, q}^{a, b}$ of our original problem has both an interior and a boundary part, we do not work directly with $L^{r}(M)$ or $L^{s}(\partial M)$ norms. Instead, we use a norm which is in a sense a combination of the two, and which reduces to those in the limit cases $a=1$, $b=0$ and $a=0, b=1$. This is defined in Lemma 2.2. Its norm properties are used in Lemma 2.5. The analogs for the boundaryless case of Lemma 2.5, Proposition 2.6 and Proposition 2.10 can be found, for example, in [LP]. 
The notation set forth by the next lemma will be used throughout this section.

Lemma 2.2. Suppose ||$_{1}$ is a norm and ||$_{2}$ is a seminorm on a vector space $W$. Let $\alpha>\beta>1$. Then

(a) For each $v \in W, v \neq 0$, there exists a unique positive number, which we denote by $|v|_{\alpha, \beta}^{a, b}$, such that

$$
a\left(|v|_{\alpha, \beta}^{a, b}\right)^{-\alpha}|v|_{1}^{\alpha}+b\left(|v|_{\alpha, \beta}^{a, b}\right)^{-\beta}|v|_{2}^{\beta}=1 .
$$

(b) ||$_{\alpha, \beta}^{a, b}$, defined to be 0 if $v=0$, is a norm on $W$.

(c) ||$_{\alpha, \beta}^{a, b}$ is equivalent to $c||_{1}+d||_{2}$ for every $c>0, d>0$.

Proof. Part (a) follows from the fact that the function $a x^{-\alpha}|v|_{1}^{\alpha}+b x^{-\beta}|v|_{2}^{\beta}$ is strictly decreasing with range $(0, \infty)$. Homogeneity of ||$_{\alpha, \beta}^{a, b}$ follows easily from its uniqueness. To show that the triangle inequality holds, suppose by contradiction that $|v+u|_{\alpha, \beta}^{a, b}>|v|_{\alpha, \beta}^{a, b}+|u|_{\alpha, \beta}^{a, b}$, for some $v \neq 0, u \neq 0$ in $W$. Then

$$
\begin{aligned}
1 & =a\left(|v+u|_{\alpha, \beta}^{a, b}\right)^{-\alpha}|v+u|_{1}^{\alpha}+b\left(|v+u|_{\alpha, \beta}^{a, b}\right)^{-\beta}|v+u|_{2}^{\beta} \\
& <a\left(\frac{|v|_{1}+|u|_{1}}{|v|_{\alpha, \beta}^{a, b}+|u|_{\alpha, \beta}^{a, b}}\right)^{\alpha}+b\left(\frac{|v|_{2}+|u|_{2}}{|v|_{\alpha, \beta}^{a, b}+|u|_{\alpha, \beta}^{a, b}}\right)^{\beta} .
\end{aligned}
$$

We will reach a contradiction with the following result, whose proof is an easy application of the method of Lagrange multipliers in $\mathbb{R}^{4}$ : If $A, B$ are positive numbers and

$$
f(x, y, z, w)=a\left(\frac{x+y}{A+B}\right)^{\alpha}+b\left(\frac{z+w}{A+B}\right)^{\beta},
$$

then $f \leq 1$ on the set $\left\{a A^{-\alpha} x^{\alpha}+b A^{-\beta} z^{\beta}=1, a B^{-\alpha} y^{\alpha}+b B^{-\beta} w^{\beta}=\right.$ $1, x, y, z, w \geq 0\}$.

We now make a definite choice of $W,||_{1}$ and ||$_{2}$. Let $\alpha \leq \frac{2 n}{n-2}, \beta \leq$ $\frac{2(n-1)}{n-2}, \alpha>\beta>1$. We take $W$ in Lemma 2.2 to be $H^{1}(M)$, and we let $|v|_{1}=\|v\|_{L^{\alpha}(M)}=\left(\int_{M}|v|^{\alpha} d \omega\right)^{\frac{1}{\alpha}},|v|_{2}=\|v\|_{L^{\beta}(\partial M)}=\left(\int_{\partial M}|v|^{\beta} d \sigma\right)^{\frac{1}{\beta}}$. 
Recall that by the Sobolev Embedding Theorem, $H^{1}(M) \longrightarrow L^{\alpha}(M)$ and $H^{1}(M) \longrightarrow L^{\beta}(\partial M)$. We denote the resulting norm, defined as in Lemma 2.2 , by ||$_{\alpha, \beta, g}^{a, b}$, where we have indicated its dependence on the underlying metric $g$, or simply by ||$_{\alpha, \beta}^{a, b}$, when no confusion may arise. We fix notation before proceeding. Throughout this paper, $K$ will stand for a generic positive constant. We will indicate in brackets the dependence of $K$ on the various relevant parameters. The notation $K^{o}(\epsilon)$ will indicate that $K \rightarrow 0$ as $\epsilon \rightarrow 0$, that is, that $K$ is $o(\epsilon)$. In the proofs we will always assume $\epsilon$ to be sufficiently small. The norms ||$_{\alpha, \beta}^{a, b}$ satisfy the following continuity property.

Lemma 2.3. Let $1<\tilde{\alpha} \leq p, 1<\tilde{\beta} \leq q$. If $\tilde{\alpha}-\epsilon \leq \alpha \leq \tilde{\alpha}$ and $\tilde{\beta}-\epsilon \leq \beta \leq \tilde{\beta}$, then for all $v$ in $H^{1}(M)$

$$
|v|_{\alpha, \beta}^{a, b} \leq\left(1+K^{o}(\epsilon)\right)|v|_{\tilde{\alpha}, \tilde{\beta}}^{a, b} .
$$

Proof. We may assume $v \not \equiv 0$. We first observe that if $x^{\tilde{\alpha}}+y^{\tilde{\beta}}=1, x \geq 0$, $y \geq 0$, then $x^{\alpha}+y^{\beta} \leq \max \left(\frac{\tilde{\alpha}}{\alpha}, \frac{\tilde{\beta}}{\beta}\right)$. From

$$
\left(a^{\frac{1}{\tilde{\alpha}}} \frac{|v|_{L^{\tilde{\alpha}}(M)}}{|v|_{\tilde{\alpha}, \tilde{\beta}}^{a, b}}\right)^{\tilde{\alpha}}+\left(b^{\frac{1}{\tilde{\beta}}} \frac{|v|_{L^{\tilde{\beta}}(\partial M)}}{|v|_{\tilde{\alpha}, \tilde{\beta}}^{a, b}}\right)^{\tilde{\beta}}=1,
$$

we obtain

$$
\begin{aligned}
& \left(a^{\frac{1}{\tilde{\alpha}}} \frac{|v|_{L^{\tilde{\alpha}}(M)}}{|v|_{\tilde{\alpha}, \tilde{\beta}}^{a, b}}\right)^{\alpha}+\left(b^{\frac{1}{\tilde{\beta}}} \frac{|v|_{L^{\tilde{\beta}}(\partial M)}}{|v|_{\tilde{\alpha}, \tilde{\beta}}^{a, b}}\right)^{\beta} \leq \max \left(\frac{\tilde{\alpha}}{\alpha}, \frac{\tilde{\beta}}{\beta}\right) \\
& \leq \max \left(\frac{\tilde{\alpha}}{\alpha}, \frac{\tilde{\beta}}{\beta}\right)\left[(a V)^{1-\frac{\alpha}{\tilde{\alpha}}}\left(a^{\frac{1}{\tilde{\alpha}}} \frac{|v|_{L^{\tilde{\alpha}}(M)}}{|v|_{\alpha, \beta}^{a, b}}\right)^{\alpha}+(b A)^{1-\frac{\beta}{\tilde{\beta}}}\left(b^{\frac{1}{\tilde{\beta}}} \frac{|v|_{L^{\tilde{\beta}}(\partial M)}}{|v|_{\alpha, \beta}^{a, b}}\right)^{\beta}\right],
\end{aligned}
$$

where we have used Hölder's Inequality. Here $V$ is the volume of $M$ and $A$ the area of $\partial M$. Choosing $K^{o}(\epsilon)$ yields the result.

For $\epsilon>0$, we let $\Lambda_{\epsilon}=\{(\alpha, \beta) \mid p-\epsilon<\alpha<p, q-\epsilon<\beta<q\}$. The next lemma bounds the $\lambda_{\alpha, \beta}$ uniformly on $\Lambda_{\epsilon}$ for $\epsilon$ small.

Lemma 2.4. (a) $\limsup _{(\alpha, \beta) \rightarrow(p, q)} Q_{\alpha, \beta}^{a, b} \leq Q_{p, q}^{a, b}$. 
(b) There exist $\epsilon>0$ and $K$ such that $\left|\lambda_{\alpha, \beta}\right| \leq K$ for $(\alpha, \beta) \in \Lambda_{\epsilon}$.

Proof. We may assume $\alpha>\beta$ for $(\alpha, \beta) \in \Lambda_{\epsilon}$. Let $\delta>0$. Let $\phi \in B_{p, q}$ such that $E(\phi)<Q_{p, q}^{a, b}+\delta$. Let $t_{\alpha, \beta}=\left(|\phi|_{\alpha, \beta}^{a, b}\right)^{-1}$. Then $Q_{\alpha, \beta}^{a, b} \leq E\left(t_{\alpha, \beta} \phi\right)=$ $t_{\alpha, \beta}^{2} E(\phi)$. If $t_{\alpha, \beta} \geq 1$, then

$$
1=a t_{\alpha, \beta}^{\alpha} \int_{M}|\phi|^{\alpha} d \omega+b t_{\alpha, \beta}^{\beta} \int_{\partial M}|\phi|^{\beta} d \sigma \geq t_{\alpha, \beta}^{\beta}\left(a \int_{M}|\phi|^{\alpha} d \omega+b \int_{\partial M}|\phi|^{\beta} d \sigma\right) .
$$

On the other hand, if $t_{\alpha, \beta} \leq 1$, then

$$
1=a t_{\alpha, \beta}^{\alpha} \int_{M}|\phi|^{\alpha} d \omega+b t_{\alpha, \beta}^{\beta} \int_{\partial M}|\phi|^{\beta} d \sigma \leq t_{\alpha, \beta}^{\beta}\left(a \int_{M}|\phi|^{\alpha} d \omega+b \int_{\partial M}|\phi|^{\beta} d \sigma\right) .
$$

By the Dominated Convergence Theorem, $a \int_{M}|\phi|^{\alpha} d \omega+b \int_{\partial M}|\phi|^{\beta} d \sigma \rightarrow$ 1 , as $(\alpha, \beta)$ tends to $(p, q)$, since $\phi \in B_{p, q}$. We conclude that $\lim \sup _{(\alpha, \beta) \rightarrow(p, q)} t_{\alpha, \beta} \leq 1$ and $\liminf _{(\alpha, \beta) \rightarrow(p, q)} t_{\alpha, \beta} \geq 1$, and part (a) follows. To prove part (b), notice that since the denominator in Equation 10 is bounded above and below, we need only bound the $Q_{\alpha, \beta}^{a, b}$. An upper bound is provided by part (a). A lower bound follows from $Q_{\alpha, \beta}^{a, b} \geq$ $-K\left(\int_{M} u_{\alpha, \beta}^{2} d \omega+\int_{\partial M} u_{\alpha, \beta}^{2} d \sigma\right)$ and $\int_{M} u_{\alpha, \beta}^{\alpha} d \omega \leq \frac{1}{a}, \int_{\partial M} u_{\alpha, \beta}^{\beta} \leq \frac{1}{b}$.

Remark: If $\tilde{g}=u^{\frac{4}{n-2}} g$, where $u$ is a smooth positive function, and $\phi$ is in $H^{1}(M)$, then it can be shown using the transformation Equations 4 that $E\left(u^{-1} \phi, \tilde{g}\right)=E(\phi, g)$. More generally, if $\Psi:(\tilde{M}, \tilde{g}) \rightarrow(M, g)$ is a diffeomorphism between the compact Riemannian manifolds with boundary $(\tilde{M}, \tilde{g})$ and $(M, g)$, and $\tilde{g}=\Psi^{*}\left(u^{\frac{4}{n-2}} g\right)$, then $E\left(\left(u^{-1} \phi\right) \circ \Psi, \tilde{g}\right)=E(\phi, g)$. Also, $\left|\left(u^{-1} \phi\right) \circ \Psi\right|_{p, q, \tilde{g}}^{a, b}=|\phi|_{p, q, g}^{a, b}$. It then follows that $Q_{p, q}^{a, b}(g)=Q_{p, q}^{a, b}(\tilde{g})$, and the existence of a smooth positive $Q_{p, q}^{a, b}(\tilde{g})$ minimizer implies the existence of a smooth positive $Q_{p, q}^{a, b}(g)$ minimizer.

Lemma 2.5. (a) Let $\phi$ be the restriction to $\mathbb{R}_{+}^{n}$ of a $C_{0}^{\infty}\left(\mathbb{R}^{n}\right)$ function. Then

$$
\left(|\phi|_{p, q, g_{1}}^{a, b}\right)^{2} \leq \frac{1}{Q_{p, q}^{a, b}\left(S_{+}^{n}\right)} \int_{\mathbb{R}_{+}^{n}}|\nabla \phi|^{2} d x
$$


where $g_{1}$ is the Euclidean metric and $d x$ is the Euclidean volume element.

(b) Suppose $\phi$ is a smooth function with support in a coordinate neighborhood of $M$ for which $\left|g_{i j}-\delta_{i j}\right|<\epsilon$, for $1 \leq i, j \leq n$. Then

$$
\left(|\phi|_{p, q, g}^{a, b}\right)^{2} \leq \frac{\left(1+K^{o}(\epsilon)\right)}{Q_{p, q}^{a, b}\left(S_{+}^{n}\right)} \int_{M}|\nabla \phi|^{2} d \omega .
$$

(c) Given $\epsilon>0$, there exists $K(\epsilon)$ such that for every $\phi \in H^{1}(M)$

$$
\left(|\phi|_{p, q, g}^{a, b}\right)^{2} \leq \frac{(1+\epsilon)}{Q_{p, q}^{a, b}\left(S_{+}^{n}\right)} \int_{M}|\nabla \phi|^{2} d \omega+K(\epsilon) \int_{M} \phi^{2} d \omega .
$$

Proof. Part (a) follows from the fact that $\mathbb{R}^{n}$ is conformal to $S_{+}^{n}$ minus a point (via stereographic projection) and that for $\phi$ a $C_{0}^{\infty}\left(\mathbb{R}^{n}\right)$ function $E\left(\phi, g_{1}\right)=\int_{\mathbb{R}_{+}^{n}}|\nabla \phi|^{2} d x$, since both the scalar and mean curvatures of $\mathbb{R}_{+}^{n}$ vanish identically. Part (b) follows at once from part (a) and continuity of the terms involved (see Section 3). Finally, part (c) follows from part (b) when we consider a partition of unity subordinate to an open cover of coordinate neighborhoods, each of which satisfies the condition stated on part (b) with $\epsilon$ replaced by a suitable $\epsilon^{\prime}$.

We are now ready to establish bounds on certain norms of the positive $Q_{\alpha, \beta}^{a, b}$ minimizers of $u_{\alpha, \beta}$.

Proposition 2.6. Suppose that $Q_{p, q}^{a, b}<Q_{p, q}^{a, b}\left(S_{+}^{n}\right)$. Let $\Lambda=\{(\alpha, \beta) \mid 2<$ $\alpha<p, 2<\beta<q, \beta<\alpha\}$ and let $u_{\alpha, \beta}$ be the positive $Q_{\alpha, \beta}^{a, b}$ minimizers, $(\alpha, \beta) \in \Lambda$. Then there exist $r>p, s>q$ and $K$ such that $\left|u_{\alpha, \beta}\right|_{L^{r}(M)} \leq K$ and $\left|u_{\alpha, \beta}\right|_{L^{s}(\partial M)} \leq K$ for all $(\alpha, \beta) \in \Lambda$ sufficiently close to $(p, q)$.

Proof. We will show that there exist $\delta>0$ and $K>0$ such that if $(\alpha, \beta) \in \Lambda$ and are sufficiently close to $(p, q)$ then $\left|w_{\alpha, \beta}\right|_{\alpha, \beta}^{a, b} \leq K$, where $w_{\alpha, \beta}=u_{\alpha, \beta}^{1+\delta}$. Part (c) of Lemma 2.2 then implies the statement of the proposition by choosing $p<r<(1+\delta) p, q<s<(1+\delta) q$. 
To prove the desired bound, we multiply Equation 9 by $u_{\alpha, \beta}^{1+2 \delta}$, where $\delta>0$ is to be chosen later. Integration by parts yields

$$
\begin{aligned}
& \frac{(1+2 \delta)}{(1+\delta)^{2}} \int_{M}\left|\nabla w_{\alpha, \beta}\right|^{2} d \omega+\frac{n-2}{4(n-1)} \int_{M} R w_{\alpha, \beta}^{2} d \omega+\frac{n-2}{2} \int_{\partial M} h w_{\alpha, \beta}^{2} d \sigma \\
& =a \alpha \lambda_{\alpha, \beta} \int_{M} w_{\alpha, \beta}^{2} u_{\alpha, \beta}^{\alpha-2} d \omega+b \beta \lambda_{\alpha, \beta} \int_{\partial M} w_{\alpha, \beta}^{2} u_{\alpha, \beta}^{\beta-2} d \sigma .
\end{aligned}
$$

We now determine an upper bound for the right hand side of the above inequality. We see from Equation 10 that $\lambda_{\alpha, \beta}$ has the same sign as $Q_{\alpha, \beta}^{a, b}$. If $Q_{p, q}^{a, b}(g)<0$, then $E(\phi)<0$ for some $H^{1}$ function $\phi$, and that implies $Q_{\alpha, \beta}^{a, b}<0$ for all $(\alpha, \beta)$ in $\Lambda$. In this case we take 0 as our upper bound. Analogously, if $Q_{p, q}^{a, b}(g) \geq 0$, then $\lambda_{\alpha, \beta} \geq 0$ for all $(\alpha, \beta)$ in $\Lambda$. We assume the latter. Then by Hölder's Inequality

$$
\begin{aligned}
& a \alpha \lambda_{\alpha, \beta} \int_{M} w_{\alpha, \beta}^{2} u_{\alpha, \beta}^{\alpha-2} d \omega+b \beta \lambda_{\alpha, \beta} \int_{\partial M} w_{\alpha, \beta}^{2} u_{\alpha, \beta}^{\beta-2} d \sigma \\
& \leq Q_{\alpha, \beta}^{a, b}\left(\frac{a \alpha\left|w_{\alpha, \beta}\right|_{L^{\alpha}(M)}^{2}\left|u_{\alpha, \beta}\right|_{L^{\alpha}(M)}^{\alpha-2}+b \beta\left|w_{\alpha, \beta}\right|_{L^{\beta}(\partial M)}^{2}\left|u_{\alpha, \beta}\right|_{L^{\beta}(\partial M)}^{\beta-2}}{a \alpha\left|u_{\alpha, \beta}\right|_{L^{\alpha}(M)}^{\alpha}+b \beta\left|u_{\alpha, \beta}\right|_{L^{\beta}(\partial M)}^{\beta}}\right) .
\end{aligned}
$$

We claim that the expression in brackets in the last term is not greater than $\left(\left|w_{\alpha, \beta}\right|_{\alpha, \beta}^{a, b}\right)^{2}$. Indeed, it is easy to check that if $c>0, d \geq 0$ and

$$
f(x, y)=\frac{a \alpha c^{2} x^{\alpha-2}+b \beta d^{2} y^{\beta-2}}{a \alpha x^{\alpha}+b \beta y^{\beta}}
$$

then on the set $\left\{a x^{\alpha}+b y^{\beta}=1, x \geq 0, y \geq 0\right\}$ we have $f \leq G^{2}$, where $G$ is the unique positive constant such that $a G^{-\alpha} c^{\alpha}+b G^{-\beta} d^{\beta}=1$. As a consequence we obtain

$$
\frac{(1+2 \delta)}{(1+\delta)^{2}} \int_{M}\left|\nabla w_{\alpha, \beta}\right|^{2} d \omega+\frac{n-2}{4(n-1)} \int_{M} R w_{\alpha, \beta}^{2} d \omega \leq \max \left(0, Q_{\alpha, \beta}^{a, b}\right)\left(\left|w_{\alpha, \beta}\right|_{\alpha, \beta}^{a, b}\right)^{2} .
$$

Let $\epsilon>0$. Lemmas 2.4 and 2.3 imply that $Q_{\alpha, \beta}^{a, b} \leq Q_{p, q}^{a, b}+\epsilon$ and $\left(\left|w_{\alpha, \beta}\right|_{\alpha, \beta}^{a, b}\right)^{2} \leq$ $(1+\epsilon)\left(\left|w_{\alpha, \beta}\right|_{p, q}^{a, b}\right)^{2}$, provided $(\alpha, \beta)$ is sufficiently close to $(p, q)$. With the aid of Lemma 2.5, and absorbing bounded quantities into generic constants, we conclude that there exists $K(\epsilon)$ such that for all $(\alpha, \beta)$ sufficiently close to 
$(p, q)$

$$
\begin{aligned}
\left(\left|w_{\alpha, \beta}\right|_{\alpha, \beta}^{a, b}\right)^{2} \leq & \frac{(1+\epsilon)^{2}\left(\max \left(0, Q_{p, q}^{a, b}\right)+\epsilon\right)(1+\delta)^{2}}{Q_{p, q}^{a, b}\left(S_{+}^{n}\right)(1+2 \delta)}\left(\left|w_{\alpha, \beta}\right|_{\alpha, \beta}^{a, b}\right)^{2} \\
& +K(\epsilon) \int_{M} w_{\alpha, \beta}^{2} d \omega+K \int_{\partial M} w_{\alpha, \beta}^{2} d \sigma .
\end{aligned}
$$

Since $Q_{p, q}^{a, b}<Q_{p, q}^{a, b}\left(S_{+}^{n}\right)$, we can find $\epsilon>0, \delta>0$ such that the first term in the right hand side of Inequality 11 can be absorbed into its left hand side. It remains to verify that the quantities $\int_{M} w_{\alpha, \beta}^{2} d \omega$ and $\int_{\partial M} w_{\alpha, \beta}^{2} d \sigma$ are uniformly bounded, but this follows from Hölder's Inequality if we choose $\delta$ such that $2(1+\delta)<p$ and $2(1+\delta)<q$.

We want to use elliptic estimates to establish bounds on the $C^{2, \gamma}$ norms $(0<\gamma<1)$ of the functions $u_{\alpha, \beta}$. For $\mu \geq 1$ and $\xi$ a non-negative integer, let $W^{\xi, \mu}(M)$ stand for the usual Sobolev spaces, $W^{\xi, \mu}(M)=\left\{f \mid\|f\|_{W \xi, \mu(M)}=\right.$ $\left.\sum_{i \leq \xi}\left(\int_{M}\left|\nabla^{i} f\right|^{\mu} d \omega\right)^{\frac{1}{\mu}}<\infty\right\}$. Suppose $\{L ; B\}$ is a regular elliptic system on $M$, where $L$ is an elliptic operator on $M$ and $B$ is the boundary operator. Let $\mu>1$. The following inequality is proved in [ADN]:

$$
K(\mu)\|u\|_{W^{2, \mu}(M)} \leq\|L u\|_{L^{\mu}(M)}+\|B u\|_{B_{\mu, \mu}^{1-\frac{1}{\mu}(\partial M)}}+\|u\|_{L^{\mu}(M)},
$$

for all $u \in W^{2, \mu}$. Here the spaces $B_{\mu, \mu}^{1-\frac{1}{\mu}}(\partial M)$ correspond to traces of $W^{1, \mu}(M)$ functions. They are special cases of more general Besov spaces $B_{\mu, \nu}^{\xi}(\mu>0, \nu>0, \xi$ real $)$. From interpolation theory for Besov spaces we have that $($ see $[\mathrm{P}])$

$$
\left(B_{\mu, \nu_{0}}^{\xi_{0}}(\partial M), B_{\mu, \nu_{1}}^{\xi_{1}}(\partial M)\right)_{\theta, \nu}=B_{\mu, \nu}^{\xi}(\partial M)
$$

for all $\mu, \nu_{0}, \nu_{1}, \nu$ positive and $\xi_{0} \neq \xi_{1}$, where $0<\theta<1$ and $\xi=(1-\theta) \xi_{0}+$ $\theta \xi_{1}$. Now let $\mu^{\prime}>\mu>1$, and choose $1-\frac{1}{\mu}<\zeta<1-\frac{1}{\mu^{\prime}}$. Let $\theta$ be defined by $1-\frac{1}{\mu}=\theta \zeta$. Note that $\frac{1-\frac{1}{\mu}}{1-\frac{1}{\mu^{\prime}}}<\theta<1$. We apply Equation 13 with $\xi_{0}=0$, $\xi_{1}=\zeta, \xi=1-\frac{1}{\mu}, \nu_{0}=\max (\mu, 2), \nu_{1}=\mu^{\prime}, \nu=\mu$ to obtain that for all $u \in W^{2, \mu^{\prime}}(M)$,

$$
\|B u\|_{B_{\mu, \mu}^{1-\frac{1}{\mu}}(\partial M)} \leq K(\theta, \mu)\|B u\|_{B_{\mu, \max (\mu, 2)}^{0}(\partial M)}^{1-\theta}\|B u\|_{B_{\mu, \mu^{\prime}}^{\zeta}(\partial M)}^{\theta} .
$$


We also have the embeddings $L^{\mu}(\partial M) \longrightarrow B_{\mu, \max (\mu, 2)}^{0}(\partial M)$ and $B_{\mu^{\prime}, \mu^{\prime}}^{1-\frac{1}{\mu^{\prime}}}(\partial M) \longrightarrow B_{\mu, \mu^{\prime}}^{\zeta}(\partial M)$. The latter holds since $\zeta<1-\frac{1}{\mu^{\prime}}$ and $1-\frac{1}{\mu^{\prime}}-\frac{n-1}{\mu^{\prime}}>\zeta-\frac{n-1}{\mu}$. Indeed, in general we have that $B_{\mu_{0}, \nu_{0}}^{\xi_{0}} \longrightarrow B_{\mu_{1}, \nu_{1}}^{\xi_{1}}$, provided $\xi_{0}>\xi_{1}$ and $\xi_{0}-\frac{n}{\mu_{0}}>\xi_{1}-\frac{n}{\mu_{1}}$, where $n$ is the dimension of the base space (see [N] and [T1]). We find that for all $u \in W^{2, \mu^{\prime}}(M)$

$$
\begin{aligned}
& K(\mu)\|u\|_{W^{2, \mu}(M)} \leq\|L u\|_{L^{\mu}(M)} \\
& \quad+K\left(\theta, \mu, \mu^{\prime}\right)\|B u\|_{L^{\mu}(\partial M)}^{1-\theta}\|\phi\|_{W^{1, \mu^{\prime}}(M)}^{\theta}+\|u\|_{L^{\mu}(M)},
\end{aligned}
$$

where $\phi$ is any $W^{1, \mu^{\prime}}$ extension of $B u$ to the whole of $M, \mu^{\prime}>\mu>1$ and $\frac{1-\frac{1}{\mu}}{1-\frac{1}{\mu^{\prime}}}<\theta<1$.

The following elementary arithmetic lemma will be useful. We omit the easy proof.

Lemma 2.7. Let $\frac{2 n}{n-2}<r_{0}<\frac{n(n+2)}{2(n-2)}$, and let $s_{0}=0$. Let $\left\{r_{i}\right\},\left\{s_{i}\right\}, i=$ $0,1, \ldots$, be two sequences recursively defined by

$$
\begin{aligned}
r_{i+1} & =\left\{\begin{array}{rr}
\frac{n(n-2) r_{i}}{n(n+2)-2(n-2) r_{i}}, & \text { if } \frac{2 n}{n-2}<r_{i}<\frac{n(n+2)}{2(n-2)} \\
r_{i} & \text { otherwise }
\end{array}\right. \\
s_{i+1} & =\left\{\begin{array}{cr}
\frac{(n-1)(n-2) r_{i}}{n(n+2)-2(n-2) r_{i}}, & \text { if } \frac{2 n}{n-2}<r_{i}<\frac{n(n+2)}{2(n-2)} \\
s_{i} & \text { otherwise }
\end{array}\right.
\end{aligned}
$$

Then

(a) $\left\{r_{i}\right\},\left\{s_{i}\right\}$ are both monotone non-decreasing.

(b) For $i \geq 1, r_{i}>\frac{2 n}{n-2}, s_{i}>\frac{2(n-1)}{n-2}$ and $n r_{i}<(n+2) s_{i}$.

(c) For some $i_{0}, \frac{2 n}{n-2}<r_{i_{0}}<\frac{n(n+2)}{2(n-2)}$ and $r_{i_{0}+1} \geq \frac{n(n+2)}{2(n-2)}$.

We will use Inequality 14 to generate a bootstrap argument that will show, with the aid of Proposition 2.6 and Lemma 2.7, that suitably high order norms of the $u_{\alpha, \beta}$ as in Proposition 2.6 are uniformly bounded. This is essentially the content of the next lemma.

Lemma 2.8. For $(\alpha, \beta)$ in $\Lambda=\{(\alpha, \beta) \mid 2<\alpha<p, 2<\beta<q, \beta<\alpha\}$, let $u_{\alpha, \beta}$ be the positive $Q_{\alpha, \beta}^{a, b}$ minimizers and let $\lambda_{\alpha, \beta}$ be as in Equation 10. Suppose that for some $\epsilon>0$ there exist positive constants $r>p, s>q, K$ and $K(r, s)$, with $n r \leq(n+2) s$, such that $\left|\lambda_{\alpha, \beta}\right| \leq K,\left|u_{\alpha, \beta}\right|_{L^{r}(M)} \leq K(r, s)$ and $\left|u_{\alpha, \beta}\right|_{L^{s}(\partial M)} \leq K(r, s)$ for all $(\alpha, \beta) \in \Lambda_{\epsilon}=\{(\alpha, \beta) \mid p-\epsilon<\alpha<$ $p, q-\epsilon<\beta<q, \beta<\alpha\}$. Then 
(i) There exists $K(r, s)$ such that $\left\|u_{\alpha, \beta}\right\|_{W^{2, \mu}(M)} \leq K(r, s)$ for all $(\alpha, \beta) \in$ $\Lambda_{\epsilon}$, where $\mu=\frac{n-2}{n+2} r$.

(ii) If $r<\frac{n(n+2)}{2(n-2)}$, then there exists $K(r, s)$ such that $\left|u_{\alpha, \beta}\right|_{L^{r^{\prime}}(M)} \leq K(r, s)$ and

$\leq K(r, s)$ for all $(\alpha, \beta) \in \Lambda_{\epsilon}$, where $r^{\prime}=\frac{n(n-2) r}{n(n+2)-2(n-2) r}$ and $s^{\prime}=$ $\frac{(n-1)(n-2) r}{n(n+2)-2(n-2) r}$.

Proof. All $(\alpha, \beta)$ in this proof will be assumed to lie in $\Lambda_{\epsilon}$. Let $\mu^{\prime}$ be defined by $\frac{1}{\mu^{\prime}}=\max \left(\frac{1}{\mu}-\frac{r-p}{n r}, \frac{p}{2 r}\right)$. Notice that $\mu^{\prime}>\mu$, since $\frac{p}{2 r}<\frac{(n+2) p}{2 n r}=\frac{n+2}{(n-2) r}=\frac{1}{\mu}$. Claim: There exists $K(r, s)$ such that

$$
\left(\int_{M}\left|\nabla u_{\alpha, \beta}^{\beta-1}\right|^{\mu^{\prime}} d \omega\right)^{\frac{1}{\mu^{\prime}}} \leq K(r, s)\left\|u_{\alpha, \beta}\right\|_{W^{2, \mu}(M)} .
$$

Proof of Claim: We split the proof into two cases. Suppose first that $\mu<n$. Let $\delta$ be defined by $\mu^{\prime} \delta=\frac{n \mu}{n-\mu}$. One can check that $\delta \geq \frac{n r}{n r-p \mu^{\prime}}>1$. Let $\delta^{\prime}$ be defined by $\frac{1}{\delta}+\frac{1}{\delta^{\prime}}=1$. By Hölder's Inequality

$$
\int_{M}\left|\nabla u_{\alpha, \beta}^{\beta-1}\right|^{\mu^{\prime}} d \omega \leq(q-1)\left(\int_{M} u_{\alpha, \beta}^{(\beta-2) \mu^{\prime} \delta^{\prime}} d \omega\right)^{\frac{1}{\delta^{\prime}}}\left(\int_{M}\left|\nabla u_{\alpha, \beta}\right|^{\frac{n \mu}{n-\mu}} d \omega\right)^{\frac{1}{\delta}} .
$$

Notice that $\frac{1}{\delta^{\prime}}=1-\frac{1}{\delta} \geq 1-\frac{n r-p \mu^{\prime}}{n r}=\frac{p \mu^{\prime}}{n r}$, hence $\mu^{\prime} \delta^{\prime} \leq \frac{n r}{p}$, and $(\beta-2) \mu^{\prime} \delta^{\prime} \leq$ $\frac{2 n r}{(n-2) p}=r$. Hölder's Inequality then implies

$$
\left(\int_{M} u_{\alpha, \beta}^{(\beta-2) \mu^{\prime} \delta^{\prime}} d \omega\right)^{\frac{1}{\delta^{\prime}}} \leq K\left(\int_{M} u^{r} d \omega\right)^{\frac{(\beta-2) \mu^{\prime}}{r}}
$$

On the other hand, we have that $W^{2, \mu}(M) \longrightarrow W^{1, n \mu /(n-\mu)}(M)$, by the Sobolev Embedding Theorem, and the claim when $\mu<n$ then follows from the hypothesis on $\left|u_{\alpha, \beta}\right|_{L^{r}(M)}$. Let us suppose now that $\mu \geq n$. By the Sobolev Embedding Theorem, $W^{2, \mu}(M) \longrightarrow W^{1, \tilde{\mu}}(M)$ for each $\tilde{\mu} \geq \mu$ if $\mu=$ $n$ and $W^{2, \mu}(M) \longrightarrow C^{1}(M)$ if $\mu>n$. In particular $W^{2, \mu}(M) \longrightarrow W^{1, r}(M)$ in either case. Since $\mu>n$, we have that $\frac{1}{\mu}<\frac{1}{r}+\frac{1}{n}=\frac{p}{2 r}+\frac{r-p}{n r}$, so that $\mu^{\prime}=\frac{2 r}{p}$. Let us choose $\delta^{\prime}=\frac{n r}{p \mu^{\prime}}=\frac{n}{2}$. Then $(\beta-2) \mu^{\prime} \delta^{\prime} \leq \frac{2 n r}{(n-2) p}=r$. Also, 
$\mu^{\prime} \delta=\frac{2 r n}{p(n-2)}=r$. The proof then follows as in the previous case. This proves the claim.

We let $L=\Delta-\frac{n-2}{4(n-1)} R$ and $B=\frac{\partial}{\partial \eta}+\frac{n-2}{2} h$. Then by Equation 9, $L u_{\alpha, \beta}=-a \alpha \lambda_{\alpha, \beta} u_{\alpha, \beta}^{\alpha-1}, B u_{\alpha, \beta}=b \beta \lambda_{\alpha, \beta} u_{\alpha, \beta}^{\beta-1}$. Notice that $\phi_{\alpha, \beta}=b \beta \lambda_{\alpha, \beta} u_{\alpha, \beta}^{\beta-1}$ is a smooth extension of $B u_{\alpha, \beta}$ to the whole of $M$. It follows from Inequality 14 that

$$
\begin{aligned}
& K(\mu)\left\|u_{\alpha, \beta}\right\|_{W^{2, \mu}(M)} \leq\left\|L u_{\alpha, \beta}\right\|_{L^{\mu}(M)} \\
& \quad+K\left(\theta, \mu, \mu^{\prime}\right)\left\|B u_{\alpha, \beta}\right\|_{L^{\mu}(\partial M)}^{1-\theta}\left\|\phi_{\alpha, \beta}\right\|_{W^{1, \mu^{\prime}}(M)}^{\theta}+\left\|u_{\alpha, \beta}\right\|_{L^{\mu}(M)}
\end{aligned}
$$

where $\frac{1-\frac{1}{\mu}}{1-\frac{1}{\mu^{\prime}}}<\theta<1$ (for simplicity, we could set $\theta=\frac{1}{2}+\frac{1}{2}\left(\frac{1-\frac{1}{\mu}}{1-\frac{1}{\mu^{\prime}}}\right)$, so that $\theta$ becomes a function of $r)$. We note that $(\beta-1) \mu^{\prime} \leq r$ and $(\beta-1) \mu \leq s$. It follows from Hölder's Inequality, the fact that $n r \leq(n+2) s$ and the Claim just proved that

$$
\left\|B u_{\alpha, \beta}\right\|_{L^{\mu}(\partial M)}^{1-\theta}\left\|\phi_{\alpha, \beta}\right\|_{W^{1, \mu^{\prime}}(M)}^{\theta} \leq K(r, s)\left(1+\left\|u_{\alpha, \beta}\right\|_{W^{2, \mu}(M)}\right)^{\theta},
$$

and so for every $\rho>0$

$$
\begin{aligned}
& \left\|B u_{\alpha, \beta}\right\|_{L^{\mu}(\partial M)}^{1-\theta}\left\|\phi_{\alpha, \beta}\right\|_{W^{1, \mu^{\prime}}(M)}^{\theta} \\
& \quad \leq \frac{K(r, s)}{\rho^{\frac{1}{1-\theta}}}+\theta \rho^{\frac{1}{\theta}}\left(1+\left\|u_{\alpha, \beta}\right\|_{W^{2, \mu}(M)}\right)=\frac{K(r, s)}{\rho^{\frac{1}{1-\theta}}}+\theta \rho^{\frac{1}{\theta}}+\theta \rho^{\frac{1}{\theta}}\left\|u_{\alpha, \beta}\right\|_{W^{2, \mu}(M)} .
\end{aligned}
$$

We choose $\rho$ so that the term $K\left(\theta, \mu, \mu^{\prime}\right) \theta \rho^{\frac{1}{\theta}}\left\|u_{\alpha, \beta}\right\|_{W^{2, \mu}(M)}$ can be absorbed by the left hand side of Inequality 15 . It remains to show that the terms $\left\|L u_{\alpha, \beta}\right\|_{L^{\mu}(M)}$ and $\left\|u_{\alpha, \beta}\right\|_{L^{\mu}(M)}$ are uniformly bounded. But this follows from Hölder's Inequality since $\mu<r$ and $(\alpha-1) \mu \leq \frac{(n+2)}{n-2} \mu=r$. This proves part (i). Now assume that $r<\frac{n(n+2)}{2(n-2)}$. This implies $\mu<\frac{n}{2}$. By the Sobolev Embedding Theorem, there are embeddings $W^{2, \mu}(M) \longrightarrow L^{\frac{n \mu}{n-2 \mu}}(M)$ and $W^{2, \mu}(M) \longrightarrow L^{\frac{(n-1) \mu}{n-2 \mu}}(\partial M)$. Part (ii) then follows from part (i), since $\frac{n \mu}{n-2 \mu}=\frac{n(n-2) r}{n(n+2)-2(n-2) r}$ and $\frac{(n-1) \mu}{n-2 \mu}=\frac{(n-1)(n-2) r}{n(n+2)-2(n-2) r}$.

The next lemma combines Lemma 2.4, Lemma 2.7 and Lemma 2.8 with Proposition 2.6 to establish bounds on higher order norms of subcritical minimizers.

Lemma 2.9. Suppose that $Q_{p, q}^{a, b}<Q_{p, q}^{a, b}\left(S_{+}^{n}\right)$. Let $\Lambda_{\epsilon}=\{(\alpha, \beta) \mid p-\epsilon<$ $\alpha<p, q-\epsilon<\beta<q, \beta<\alpha\}$ and let $u_{\alpha, \beta}$ be the positive $Q_{\alpha, \beta}^{a, b}$ minimizers, 
$(\alpha, \beta) \in \Lambda_{\epsilon}$. Then there exists $\epsilon>0$ such that for each $\mu>1$ there exists $K(\mu)$ such that $\left\|u_{\alpha, \beta}\right\|_{W^{2, \mu}(M)} \leq K(\mu)$, for all $(\alpha, \beta)$ in $\Lambda_{\epsilon}$.

Proof. The statement of the lemma is true for some $\mu_{0} \geq \frac{n}{2}$. This follows easily from Lemma 2.8 and Lemma 2.7. By the Sobolev Embedding Theorem, $W^{2, \mu_{0}}(M) \longrightarrow L^{r}(M), W^{2, \mu_{0}}(M) \longrightarrow L^{s}(\partial M)$, for each $r \geq 1$, $s \geq 1$. Given $\mu>\frac{n}{2}$, we choose $r, s$ such that $p<r=\frac{n+2}{n-2} \mu, s>q$ and $n r \leq(n+2) s$. Applying part (i) of Lemma 2.8 with the values of $r$ and $s$ just chosen completes the argument. The result for $1<\mu \leq \frac{n}{2}$ follows from applying Hölder's inequality.

We recall a result from Schauder elliptic theory. Suppose $\{L ; B\}$ is a regular elliptic system on $M$ and $u$ is a $C^{2, \gamma}(M)$ solution of $L u=f$ on $M$ and $B u=\phi$ on $\partial M$, where $f$ is in $C^{0, \gamma}(M)$ and $\phi$ is a globally defined function in $C^{1, \gamma}(M)$. Then there exists $K(\gamma)$ such that

$$
\|u\|_{C^{2, \gamma}(M)} \leq K(\gamma)\left(\|u\|_{C^{0}(M)}+\|f\|_{C^{0, \gamma}(M)}+\|\phi\|_{C^{1, \gamma}(M)}\right) .
$$

For a proof of the above result, see $[\mathrm{GT}]$. Inequality 16 will be useful in the proof the next proposition.

Proposition 2.10. Suppose that $Q_{p, q}^{a, b}<Q_{p, q}^{a, b}\left(S_{+}^{n}\right)$. Let $\Lambda_{\epsilon}=\{(\alpha, \beta) \mid p-$ $\epsilon<\alpha<p, q-\epsilon<\beta<q, \beta<\alpha\}$ and let $u_{\alpha, \beta}$ be the positive $Q_{\alpha, \beta}^{a, b}$ minimizers, $(\alpha, \beta) \in \Lambda_{\epsilon}$. Then there exists $\epsilon>0$ such that for each $0<\gamma<1$ there exists $K(\gamma)$ such that $\left\|u_{\alpha, \beta}\right\|_{C^{2, \gamma}(M)} \leq K(\gamma)$, for all $(\alpha, \beta)$ in $\Lambda_{\epsilon}$.

Proof. The statement of the proposition holds with the $C^{1, \gamma}(M)$ norm replacing the $C^{2, \gamma}(M)$ norm, since by the Sobolev Embedding Theorem $W^{2, \mu}(M) \longrightarrow C^{1, \gamma}(M)$ if $0<\gamma \leq 1-\frac{n}{\mu}$. The result then follows from Lemma 2.9, choosing $\mu$ large enough. To complete the proof, we make use of Inequality 16, with $L=\Delta-\frac{n-2}{4(n-1)} R, B=\frac{\partial}{\partial \eta}+\frac{n-2}{2} h, u=u_{\alpha, \beta}$, $f=a \alpha \lambda_{\alpha, \beta} u_{\alpha, \beta}^{\alpha-1}$ and $\phi=b \beta \lambda_{\alpha, \beta} u_{\alpha, \beta}^{\beta-1}$.

Proof of Theorem 2.1 Let $u_{\alpha, \beta}, \epsilon>0, \Lambda_{\epsilon}, 0<\gamma<1$ and $K(\gamma)$ be as in Proposition 2.10. The fact that $\left\|u_{\alpha, \beta}\right\|_{C^{2, \gamma}(M)} \leq K(\gamma)$ for all $(\alpha, \beta) \in \Lambda_{\epsilon}$ and the compactness of the embedding $C^{2}(M) \longrightarrow C^{2, \gamma}(M)$ imply that a sequence of the $u_{\alpha_{i}, \beta_{i}}$, with $\alpha_{i} \rightarrow p, \beta_{i} \rightarrow q$, converges to some $C^{2}$ function $u$ in the $C^{2}(M)$ topology. Since the $\lambda_{\alpha_{i}, \beta_{i}}$ are uniformly bounded, we may 
assume that $\lambda_{\alpha_{i}, \beta_{i}} \rightarrow \lambda$, for some $\lambda$. The function $u$ then satisfies the limiting equation

$$
\left\{\begin{array}{lr}
\Delta u-\frac{n-2}{4(n-1)} R u+a p \lambda u^{p-1}=0 & \text { on } M, \\
\frac{\partial u}{\partial \eta}+\frac{n-2}{2} h u=b q \lambda u^{q-1} & \text { on } \partial M .
\end{array}\right.
$$

Multiplying the above equation by $u$ and integrating by parts yields $E(u)=$ $\lim _{i \rightarrow \infty} Q_{\alpha_{i}, \beta_{i}} \leq Q_{p, q}^{a, b}$, where we have used Equation 10 and Lemma 2.4. On the other hand, $u \in B_{p, q}^{a, b}$, as $u_{\alpha_{i}, \beta_{i}} \rightarrow u$ uniformly and $u_{\alpha_{i}, \beta_{i}} \in B_{\alpha_{i}, \beta_{i}}^{a, b}$. Hence $E(u) \leq Q_{p, q}^{a, b}$ implies $E(u)=Q_{p, q}^{a, b}$, and $u$ is a nonnegative $Q_{p, q}^{a, b}$ minimizer.

\section{A priori estimates and the case $a=0, b=1$.}

Let $g_{0}$ be a fixed smooth Riemannian metric on $M$. In this section we address the issue of compactness of the set of all $Q_{p, q}^{a, b}(g)$ minimizers when $g$ varies on a sufficiently small $C^{2, \gamma}$ neighborhood of $g_{0}$, provided $Q_{p, q}^{a, b}\left(g_{0}\right)<Q_{p, q}^{a, b}\left(S_{+}^{n}\right)$. We also treat existence and compactness of minimizers in the case when $a=0$ and $b=1$. As the proofs are straightforward, we will limit ourselves to stating the main results and giving some indication on how to prove them. This includes stating a few preparatory lemmas whose proofs are left to the reader. As a matter of notation, we will use $C^{k}(\mathcal{T})\left(\operatorname{resp} . C^{k, \gamma}(\mathcal{T})\right)$ for the space of $C^{k}$ (resp. $C^{k, \gamma}$ ) symmetric $(2,0)$ tensor fields.

We first show that $Q_{p, q}^{a, b}$ is continuous in the $C^{2}(\mathcal{T})$ topology. This is Proposition 3.4. Before that we state a continuity lemma that was also used in the proof of Lemma 2.5. We use the following notation: we let $B_{p, q, g}^{a, b}$ and $E(u, g)$ be as defined by Equations 5 and 6 , respectively, and we let $B_{p, q, 0}^{a, b}$ and $E(u, 0)$ correspond to the base metric $g_{0}$. Also, we let ||$_{p, q, g}^{a, b}$ be the norm defined in Lemma 2.2, with ||$_{1}=\|\|_{L^{p}(M)}$ and ||$_{2}=\|\|_{L^{q}(\partial M)}$, where these norms now depend on the metric $g$ (but are all equivalent). We will use ||$_{p, q, 0}^{a, b}$ when $g=g_{0}$. In general we will not indicate the dependence of geometric quantities on the background metric, except for a naught subscript when they refer to the base metric $g_{0}$. The next lemma says that the $|u|_{p, q, g}^{a, b}$ are close to the $|u|_{p, q, 0}^{a, b}$, provided that $g$ is close to $g_{0}$.

Lemma 3.1. If $\left|g-g_{0}\right|_{C^{0}(\mathcal{T})}<\epsilon$ then

$$
\left(1-K^{o}(\epsilon)\right)|u|_{p, q, 0}^{a, b} \leq|u|_{p, q, g}^{a, b} \leq\left(1+K^{o}(\epsilon)\right)|u|_{p, q, 0}^{a, b},
$$

for all $u$ in $H^{1}(M)$.

We remark that $K^{o}(\epsilon)$ in Lemma 3.1 does not depend on $g$. 
Lemma 3.2. (a) If $\left|g-g_{0}\right|_{C^{2}(\mathcal{T})}<\epsilon$ and $u \in B_{p, q, g}^{a, b}$ then

$$
|E(u, g)-E(\tilde{u}, 0)| \leq K^{o}(\epsilon)\left(\int_{M}\left|\nabla_{0} \tilde{u}\right|_{0}^{2} \omega_{0}+\int_{M} \tilde{u}^{2} d \omega_{0}+\int_{\partial M} \tilde{u}^{2} d \sigma_{0}\right),
$$

where $\tilde{u}=\left(|u|_{p, q, 0}^{a, b}\right)^{-1} u$.

(b) There exists $K$ such that for all $u$ in $B_{p, q, 0}^{a, b}$,

$$
\int_{M}\left|\nabla_{0} u\right|_{0}^{2} \omega_{0}+\int_{M} u^{2} d \omega_{0}+\int_{\partial M} u^{2} d \sigma_{0} \leq E(u, 0)+K .
$$

Observe that $\tilde{u}$ in part (a) belongs to $B_{p, q, 0}^{a, b}$. Also, since $|u|_{p, q, g}^{a, b}=1$, Lemma 3.1 implies that $1-K^{o}(\epsilon) \leq\left(|u|_{p, q, 0}^{a, b}\right)^{-1} \leq 1+K^{o}(\epsilon)$. The proof of part (a) then follows from the fact that the scalar and mean curvatures depend on derivatives of the metric up to second order. To prove part (b), we just need to use that, by Proposition 2.3 in [E4], given $\varepsilon>0$ there exists $K(\varepsilon)$ such that $\int_{M} u^{2} d \omega_{0} \leq \varepsilon \int_{M}\left|\nabla_{0} u\right|_{0}^{2} d \omega_{0}+K(\varepsilon)$ and $\int_{\partial M} u^{2} d \sigma_{0} \leq \varepsilon \int_{M}\left|\nabla_{0} u\right|_{0}^{2} d \omega_{0}+K(\varepsilon)$. By combining parts (a) and (b) of Lemma 3.2 we obtain the following corollary.

Corollary 3.3. If $\left|g-g_{0}\right|_{C^{2}(\mathcal{T})}<\epsilon$ and $u \in B_{p, q, g}^{a, b}$ then

$$
|E(u, g)-E(\tilde{u}, 0)| \leq K^{o}(\epsilon)(E(\tilde{u}, 0)+1),
$$

where $\tilde{u}=\left(|u|_{p, q, 0}^{a, b}\right)^{-1} u$.

The continuity of $Q$ follows at once from Corollary 3.3. We state it below.

Proposition 3.4. The functional $g \rightarrow Q_{p, q}^{a, b}(g)=\inf \left\{E(u, g) \mid u \in B_{p, q, g}^{a, b}\right\}$ is continuous in the $C^{2}(\mathcal{T})$ topology.

We let

$$
\begin{aligned}
& D_{\epsilon, \gamma}:=\left\{g \mid g \text { is a smooth Riemannian metric and }\left|g-g_{0}\right|_{C^{2, \gamma}(\mathcal{T})}<\epsilon\right\} \\
& \mathfrak{D}_{\epsilon, \gamma}^{a, b}:=\left\{u \mid u \text { is a smooth positive } Q_{p, q}^{a, b}(g) \text { minimizer, with } g \in D_{\epsilon, \gamma}\right\} .
\end{aligned}
$$

The first main result of this section is the following.

Theorem 3.5. Let $g_{0}$ be a smooth Riemannian metric such that $Q_{p, q}^{a, b}(g)<$ $Q_{p, q}^{a, b}\left(S_{+}^{n}\right)$. Then, given $0<\gamma<1$, there exist $\epsilon=\epsilon(\gamma)>0$ and $K(\gamma)$ such that $\|u\|_{C^{2, \gamma}(M)} \leq K(\gamma)$ for all $u$ in $\mathfrak{D}_{\epsilon, \gamma}^{a, b}$. 
This is analogous to Proposition 2.10 and the proof follows a similar path: we need the (locally uniform) counterparts to Lemmas 2.4 (part (b)) and 2.5, Proposition 2.6, and Lemmas 2.8 and 2.9. These are all easy to show. Observe that by continuity of $Q_{p, q}^{a, b}(g)$ at $g_{0}$ in the $C^{2}(\mathcal{T})$ topology we may assume that $Q_{p, q}^{a, b}\left(g_{0}\right)-\epsilon^{\prime}<Q_{p, q}^{a, b}(g)<Q_{p, q}^{a, b}\left(g_{0}\right)+\epsilon^{\prime}<Q_{p, q}^{a, b}\left(S_{+}^{n}\right)$, for some $\epsilon^{\prime}>$ 0 , and for all $g$ is in $D_{\epsilon, \gamma}$, provided $Q_{p, q}^{a, b}\left(g_{0}\right)<Q_{p, q}^{a, b}\left(S_{+}^{n}\right)$. The counterparts to Lemmas 2.8 and 2.9 use locally uniform versions of Inequalities 14 and 16 , which involve a family of elliptic systems depending on the background metric. These inequalities in turn again follow easily from the fact that the scalar curvature and mean curvature involve derivatives of the metric up to second order.

We now turn to the case when $a=0$ and $b=1$. We define

$$
B_{q, g}^{0,1}=\left\{u \in H^{1}(M) \mid \int_{\partial M} u^{q} d \sigma=1\right\} .
$$

Nonnegative critical points of the functional $E$ (as defined by Equation 6) restricted to $B_{q, g}^{0,1}$ correspond to (necessarily smooth) solutions of

$$
\left\{\begin{array}{lr}
\Delta u-\frac{n-2}{4(n-1)} R u=0 & \text { on } M \\
\frac{\partial u}{\partial \eta}+\frac{n-2}{2} h u=E(u, g) u^{q-1} & \text { on } \partial M
\end{array}\right.
$$

Existence of positive critical points was proved by Escobar ([E2]) for a large class of manifolds. If $u$ is a smooth positive solution of Equation 18 then $\tilde{g}=u^{\frac{4}{n-2}} g$ is a smooth scalar flat Riemannian metric with constant mean curvature $\tilde{h}=\frac{2}{n-2} E(u, g)$. If we let

$$
Q_{q}^{0,1}(g)=\inf \left\{E(u, g) \mid u \in H^{1}(M), u \in B_{q, g}^{0,1}\right\},
$$

then $u \in B_{q, g}^{0,1}$ will be called a $Q_{q}^{0,1}(g)$ minimizer if $E(u, g)=Q_{q}^{0,1}(g) \cdot Q_{q}^{0,1}(g)$ minimizers are smooth and do not change sign. We always have $Q_{q}^{0,1}(g) \leq$ $Q_{q}^{0,1}\left(S_{+}^{n}\right)([\mathrm{E} 2])$, but it is possible to have $Q_{q}^{0,1}(g)=-\infty([\mathrm{E} 3])$. More generally, for $2 \leq \beta \leq q$, we let

$$
B_{\beta, g}^{0,1}(g)=\left\{u \in H^{1}(M) \mid \int_{\partial M} u^{\beta} d \sigma=1\right\},
$$

and

$$
Q_{\beta}^{0,1}(g)=\inf \left\{E(u, g) \mid u \in H^{1}(M), u \in B_{\beta, g}^{0,1}\right\} .
$$


We will say that $u$ is a $Q_{\beta}^{0,1}(g)$ minimizer if $u \in B_{\beta, g}^{0,1}$ and $E(u, g)=B_{\beta, g}^{0,1}$. Nonnegative $Q_{\beta}^{0,1}(g)$ minimizers are (necessarily smooth and positive) solutions of

$$
\left\{\begin{array}{lr}
\Delta u-\frac{n-2}{4(n-1)} R u=0 & \text { on } M \\
\frac{\partial u}{\partial \eta}+\frac{n-2}{2} h u=Q_{\beta}^{0,1}(g) u^{\beta-1} & \text { on } \partial M .
\end{array}\right.
$$

It follows from Hölder's Inequality that either $0 \leq Q_{q}^{0,1}(g) \leq Q_{\beta}^{0,1}(g) \leq$ $Q_{2}^{0,1}(g)$ or $-\infty \leq Q_{2}^{0,1}(g) \leq Q_{\beta}^{0,1}(g) \leq Q_{q}^{0,1}(g)<0$, for all $2 \leq \beta \leq q$. When $-\infty<Q_{2}^{0,1}(g)$ there exists a positive $Q_{2}^{0,1}(g)$ minimizer $v$. The metric $\hat{g}=v^{\frac{4}{n-2}} g$ is a scalar flat metric whose mean curvature either vanishes identically or does not change sign. It is then possible to show, using the compactness of the embeddings $H^{1}(M) \hookrightarrow L^{\beta}(\partial M)$, that positive $Q_{\beta}^{0,1}(\hat{g})$ minimizers exist for $2 \leq \beta<q$. We remark that a $Q_{q}^{0,1}(g)$ minimizer exists if and only if a $Q_{q}^{0,1}(\hat{g})$ minimizer exists (see Remark preceeding Lemma 2.5).

Proposition 3.6. If $-\infty<Q_{2}^{0,1}(g)$ and $Q_{q}^{0,1}(g)<Q_{q}^{0,1}\left(S_{+}^{n}\right)$ then there exists a positive $Q_{q}^{0,1}(g)$ minimizer.

Existence of critical points is established in [E2]. Below we indicate how to show those are indeed minimizers. We may assume that $g$ is a scalar flat metric whose mean curvature either vanishes identically or does not change sign. We will denote by $u_{\beta}$ a positive $Q_{\beta}^{0,1}(g)$ minimizer. The proof of Proposition 3.6 follows the same steps as the proof of Theorem 2.1. The only significant difference occurs in the proof of the counterpart to Proposition 2.6, for now we must make use of the fact that the scalar curvature vanishes identically in order to obtain the desired estimates. Specifically, we can prove the following.

Lemma 3.7. Suppose that $Q_{q}^{0,1}(g)<Q_{q}^{0,1}\left(S_{+}^{n}\right)$ and that the scalar curvature of $g$ vanishes identically. Let $u_{\beta}$ be the smooth positive $Q_{\beta}^{0,1}(g)$ minimizers, $2<\beta<q$. There exist positive constants $r>p, s>q$ and $K$ such that $\left|u_{\beta}\right|_{L^{r}(M)} \leq K$ and $\left|u_{\beta}\right|_{L^{s}(\partial M)} \leq K$ for all $\beta<q$ sufficiently close to $p$.

As already mentioned, the proof of Proposition 3.6 then follows in a way similar to that of Theorem 2.1, with Lemma 3.7 replacing Proposition 2.6. We now want to show that a result similar to Theorem 3.5 holds when $a=0$, $b=1$. We first point out that both $Q_{2}^{0,1}$ and $Q_{q}^{0,1}$ satisfy some continuity properties in the $C^{2}(\mathcal{T})$ topology. 
Lemma 3.8. Suppose that $g_{0}$ is a $C^{2}$ metric whose scalar curvature $R_{0}$ vanishes identically. Then $Q_{2}^{0,1}$ and $Q_{q}^{0,1}$ are continuous at $g_{0}$ in the $C^{2}(\mathcal{T})$ topology.

In the proof we need the following version of part (b) of Lemma 3.2: There exists $K$ such that for all $u \in H^{1}(M)$ such that either $\int_{\partial M}|u|^{q} d \sigma_{0}=1$ or $\int_{\partial M}|u|^{2} d \sigma_{0}=1$

$$
\int_{M}\left|\nabla_{0} u\right|_{0}^{2} \omega_{0}+\int_{M} u^{2} d \omega_{0}+\int_{\partial M} u^{2} d \sigma_{0} \leq K(E(u, 0)+1) .
$$

Lemma 3.9. Suppose that $g_{0}$ is a smooth metric with $Q_{2}^{0,1}\left(g_{0}\right)>-\infty$. Then

(a) $Q_{q}^{0,1}$ is continuous at $g_{0}$ in the $C^{2}(\mathcal{T})$ topology.

(b) There exists a $C^{2}(\mathcal{T})$ neighborhood of $g_{0}$ where $Q_{2}^{0,1}$ is finite.

To prove the above result, we let $v$ be a smooth positive function such that $v^{\frac{4}{n-2}} g_{0}$ has vanishing scalar curvature and consider the map $g \rightarrow \varphi(g)=$ $v^{\frac{4}{n-2}} g$. Then $\varphi$ is a homeomorphism of $C^{2}(\mathcal{T})$ which leaves $Q_{q}^{0,1}$ invariant (see Remark before Lemma 2.5). Lemma 3.8 then implies part (a). Since finiteness of $Q_{2}^{0,1}$ is a conformal invariant, part (b) follows.

We now fix a smooth metric $g_{0}$. We let $D_{\epsilon, \gamma}$ be as before and we let $\mathfrak{D}_{\epsilon, \gamma}^{0,1}:=\left\{u \mid u\right.$ is a smooth positive $Q_{q}^{0,1}(g)$ minimizer, with $\left.g \in D_{\epsilon, \gamma}\right\}$. We have the following analogue to Theorem 3.5.

Proposition 3.10. (a) Let $g_{0}$ be a smooth, scalar flat Riemannian metric such that $Q_{q}^{0,1}\left(g_{0}\right)<Q_{q}^{0,1}\left(S_{+}^{n}\right)$. Then, given $0<\gamma<1$, there exist $\epsilon=\epsilon(\gamma)>0$ and $K(\gamma)$ such that $\|u\|_{C^{2, \gamma}(M)} \leq K(\gamma)$ for all $u$ in $\mathfrak{D}_{\epsilon, \gamma}^{0,1}$.

(b) The conclusion of part (a) remains true if we replace the scalar flat condition by the requirement that $Q_{2}^{0,1}\left(g_{0}\right)>-\infty$.

Proof. Part (b) follows from part (a) by considering the map $g \rightarrow \varphi(g)=$ $v^{\frac{4}{n-2}} g$ as above. The proof of part (a) follows the same steps as the proof of Theorem 3.5. The only significant difference occurs in the proof of the locally uniform counterpart to Proposition 2.6. This is because we do not have $R \equiv 0$ for all metrics in a neighborhood of $g_{0}$. However, we do have $R \leq K^{o}(\epsilon)$, since $R_{0} \equiv 0$, and it is easy to verify that this suffices. 
Acknowledgements: This work contains results from the author's Ph.D. dissertation $([\mathrm{Ar}])$. The author's doctorate studies at Cornell University were partially funded by CNPq-Brazil. The author wishes to thank his Ph.D. adviser, Prof. José F. Escobar, for his help and support. During the writing of this paper the author was a guest at the Departamento de Matemática at Universidade Federal de Pernambuco, Recife, Brazil, and was partially supported by CNPq, Pronex \#41.96.0860.00.

\section{References.}

[ADN] S. Agmon, A. Douglis and L. Nirenberg, Estimates near the boundary for solutions of elliptic partial differential equations satisfying general boundary conditions I, Comm. Pure Appl. Math. 12 (1959), $623-727$.

[Ar] H. Araújo, On the Total Scalar Curvature plus Total Mean Curvature Functional, Ph.D. thesis, Cornell University, 2001.

[Au] T. Aubin, Équations différentielles non linéaires et problème de Yamabe concernant la courbure scalaire, J. Math. Pures Appl. (9) 55 (1976), no. 3, 269-296.

[C] P. Cherrier, Problèmes de Neumann non linéaires sur les variétés riemanniennes, C. R. Acad. Sci. Paris Sér. I Math. 292 (1981), noi3, 637-640.

[E1] J.F. Escobar, The Yamabe problem on manifolds with boundary, J. Differential Geom. 35 (1992), no. 1, 21-84.

[E2] J.F. Escobar, Conformal deformation of a Riemannian metric to a scalar flat metric with constant mean curvature on the boundary, Ann. of Math. (2) 136 (1992), no. 1, 1-50.

[E3] J.F. Escobar, Addendum: "Conformal deformation of a Riemannian metric to a scalar flat metric with constant mean curvature on the boundary", Ann. of Math. (2) 139 (1994), no. 3, 749-750.

[E4] J.F. Escobar, Conformal deformation of a Riemannian metric to a constant scalar curvature metric with constant mean curvature on the boundary, Indiana Univ. Math. J. 45 (1996), no. 4, 917-943. 
[GT] D. Gilbarg and N. Trudinger, Elliptic Partial Differential Equations of Second Order, $2^{\text {nd }}$ ed., Grundlehren der Mathematischen Wissenschaften, 224, Springer-Verlag, Berlin-New York, 1983.

[HL1] Z.C. Han and Y.Y. Li, The Yamabe problem on manifolds with boundary: existence and compactness results, Duke Math. J. 99 (1999), no. 3, 489-542.

[HL2] Z.C. Han and Y.Y. Li, The existence of conformal metrics with constant scalar curvature and constant boundary mean curvature, Comm. Anal. Geom. 8 (2000), no. 4, 809-869.

[LP] J. Lee and T. Parker, The Yamabe problem, Bull. Amer. Math. Soc. (N.S.) 17 (1987), no. 1, 37-91.

[LM] J.L. Lions and E. Magenes, Non-homogeneous Boundary Value Problems and Applications, Volume I, Translated from the French by P. Kenneth, Die Grundlehren der mathematischen Wissenschaften in Einzeldarstellungen, Band 181, Springer-Verlag, New York-Heidelberg, 1972.

[N] S.M. Nikol'ski, Approximation of Functions of Several Variables and Inbedding theorems, translated from the Russian by J.M. Danskin, Die Grundlehren der mathematischen Wissenschaften in Einzeldarstellungen, Band 205, Springer-Verlag, Berlin-Heidelberg, 1975 .

[P] J. Peetre, Sur les espaces de Besov, C.R. Acad. Sci. Paris Sér. $A-B 264$ (1967), 281-283.

[S] R. Schoen, Conformal deformation of a Riemannian metric to constant scalar curvature, J. Differential Geom. 20 (1984), no. 2, 479-495.

[T1] H. Triebel Theory of Function Spaces, Monographs in Mathematics, 78. Birkhäuser Verlag, Basel-Boston, Mass., 1983.

[T2] H. Triebel Interpolation Theory, Function Spaces, Differential Operators, $2^{\text {nd }}$ ed., Johann Ambrosius Barth, Heidelberg, 1995.

[T] N. Trudinger, Remarks concerning the conformal deformation of Riemannian structures on compact manifolds, Ann. Scuola Norm. Sup. Pisa (3) 221968 265-274. 
[Y] H. Yamabe On a deformation of Riemannian structures on compact manifolds, Osaka Math. J. 121960 21-37.

Departamento de Matemática

Universidade Federal de Pernambuco

RECIFE, PE, CEP 50740-540, BRAZIL

henrique@dmat.ufpe.br

Received August 2001

REVISED MARCH 2002. 